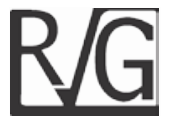

\title{
Cambio estructural y desindustrialización: evidencia para Colombia
}

\author{
Pérez Fuentes, Dewin ${ }^{*}$ \\ Ahumada Lagares, Gustavo*
}

\section{Resumen}

Este artículo tiene como objetivo analizar el cambio estructural de la industria manufacturera colombiana, durante el período 1970-2008. Para tal fin, se examinan los determinantes del crecimiento industrial desde el enfoque de las fuentes de demanda, con base en la metodología desarrollada por Chenery y Syrquin (1989). El estudio se enfoca en el período de ajuste de los años noventa, para determinar los efectos de la aplicación del modelo de liberalización económica, en la dinámica, transformaciones y perspectivas de crecimiento de la industria manufacturera frente a la competencia externa que instaura el proceso de internacionalización de la economía. Los resultados obtenidos de la implementación de un índice de cambio estructural y de un modelo econométrico de datos panel, indican la existencia de un cambio estructural con un sesgo desindustrializador a partir de mediados de la década de los años setenta, cuya principal característica es la pérdida progresiva de la generación de valor agregado de la industria manufacturera.

Palabras clave: cambio estructural; desindustrialización; producción doméstica de bienes industriales.

Recibido: 12-07-14 Aceptado: 20-07-15

* Magister en Ciencias Económicas de la Universidad Nacional de Colombia, estudiante del Doctorado en Ciencias Económicas de la Universidad del Zulia. Docente y Director del programa de economía de la Universidad de Cartagena, Director del Observatorio del Mercado Laboral de Cartagena y Bolívar, Colombia. E-mail: dperezf@unicartagena.edu.co; dewinperez@yahoo.es

** Economista de la Universidad de Cartagena. Asistente de Investigación del Observatorio del Caribe Colombiano, Colombia. E-mail: gusahu18@gmail.com 


\title{
Structural Change and deindustrialization: Evidence for Colombia
}

\begin{abstract}
This research is aimed to analyze the structural change of the Colombian manufacturing industry during the 1970-2008 period. For such purpose, the industrial growth determinants from the demand sources approach are examined based on the methodology developed by Chenery and Syrquin (1989). The study focuses on the nineties adjustment period in order to determine the effects of the economic liberalization model application on the dynamic, transformation and growing perspectives of the manufacturing industries facing the external competence which establishes the economy internationalization process. The obtained results from the implementation of a structural change index and an econometric panel data model indicate the existence of a structural change with a deindustrialization bias starting from the mid seventies whose main characteristic is the progressive loss of the manufacturing industry added value.
\end{abstract}

Key words: structural change; deindustrialization; domestic industrial goods production.

\section{Introducción}

Desde la década de los años setenta la industria colombiana estancó su proceso de transformación productiva, escaso de diversificación, encadenamientos productivos y avances tecnológicos, impidiendo el avance hacia estructuras industriales que permitan procesos de desarrollo industrial (García, 2005:14).

El bajo ritmo de crecimiento del sector manufacturero, el estancamiento relativo de la diversificación productiva y el agotamiento tecnológico; la transición hacia una trayectoria de deterioro $y$ de débil transformación del patrón de crecimiento, indican la reducción de la participación del sector manufacturero en la estructura productiva y evidencia.

El análisis del cambio estructural en el contexto del desarrollo y el crecimiento económico agregado tiene una larga tradición y se remonta a la literatura clásica del desarrollo económico (Chenery, 1982; Chenery, 1986; Prebisch, 1986; Vernon, 1998). Sin embargo, este es un tema que aún prevalece en la investigación económica actual, como han señalado Silva y Teixeira (2008). En su estudio de la cuestión de los cambios estructurales, abarcan la historia de esta rama de la investigación y sus múltiples enfoques y facetas diferentes. Por su parte, Thirwall (2003) y Cimoli et al (2011) coinciden en la existencia de una relación directa entre transformación industrial, capacidad tecnológica y crecimiento económico, sustentada en la acumulación de hechos estilizados en procesos de crecimiento económico a través de la evidencia mostrada en las diferentes estructuras económicas y procesos de inversión para el desarrollo de bienes de capital por parte de las economías industrializadas.

En Colombia autores como Garay (1998), García (2002) y Misas (2002), concluyen que el desmonte del modelo de sustitución de importaciones llevó a un estancamiento del sector manufacturero y a un progresivo proceso de desindustrialización, bajos niveles de encadenamientos productivos y el 
escaso impulso de la economía en su conjunto. Sumado a lo anterior, un hecho de mucha incidencia en la evolución de la industria en los años siguientes fue la implementación del modelo de apertura económica a inicios de 1990; en este aspecto Echavarria (2001) considera que, una vez implementado el cambio de modelo económico, han existido sectores ganadores de competitividad como el sector minero. Para el autor, la pérdida de dinamismo del sector manufacturero a nivel agregado se debió a una mala implementación del consenso de Washington.

Por otra parte Ortiz et al (2009) afirman que desde finales de la década de 1970 la industria manufacturera entra en un proceso de estancamiento, el cual se refuerza con la apertura económica de inicios de la década de 1990. Posteriormente, Moncayo (2011) argumenta que el proceso de industrialización colombiano ha sido relativamente débil o mediocre, la manufactura impulsó el crecimiento desde la década de 1930 hasta 1970, y el proceso de estancamiento industrial fue agravado por las reformas estructurales de la década de 1990.

El objetivo del artículo es analizar el cambio estructural en la industria manufacturera colombiana, durante el período 1970-2008. Para este propósito, se estudian variables como el producto interno bruto nacional, producto interno bruto manufacturero, exportaciones e importaciones, demanda doméstica de bienes industriales, el coeficiente de apertura externa, entre otras variables relacionadas con el sector manufacturero. La información fue tomada de la Encuesta Anual Manufacturera (EAM) y las cuentas nacionales del Departamento Administrativo Nacional de Estadísticas (DANE) para el periodo 1970-2008. No es posible analizar series de datos más recientes, pues los cambios metodológicos no permiten hacer compatible las cifras históricas con datos actuales; además, el estudio se concentra en el periodo de ajuste (década de los noventa).

Se emplea la metodología de datos de panel, debido a la bondad en la unión de cortes transversales que permite medir efectos no observables en muestras de este tipo de datos, y mejoran la precisión de las estimaciones (Baltagi, 2005:4). El esquema de modelación seleccionado se especificó entre efectos fijos $y$ efectos aleatorios ${ }^{1}$. Se realizó la prueba de Breusch y Pagan para identificar problemas de correlación contemporánea en los residuos.

\section{Crecimiento de la producción industria manufacturera: un análisis desde la perspectiva de las fuentes de demanda}

La dinámica del cambio de la productividad y el crecimiento industrial en el país han utilizado la función de producción neoclásica con retornos constantes a escala, del tipo CobbDouglas, aunque este enfoque tiene muchas críticas del orden conceptual y empírico, debido a los problemas teóricos

1 Se llevó a cabo una prueba de Hausman para determinar la mejor estimación entre el modelo de efectos fijos y el de efectos aleatorios, el resultado de esta prueba arrojó que se debe rechazar la hipótesis nula de ortogonalidad (no correlación entre los regresores y el término de error, es decir, que $E\left[\varepsilon_{i t} \mid X_{i t}=0\right]$ al $5 \%$ de significancia, por lo tanto el estimador de efectos aleatorios es la mejor opción para estimar el panel. 
que subyacen en la función de producción agregada (Maldonado, 2010:52). Por otra parte la existencia de otros desarrollos teóricos sobresalientes busca establecer las relaciones de causalidad de la demanda hacia el crecimiento y la productividad. Estas aproximaciones teóricas se fundamentan en las leyes de Kaldor-Verdoorn, referenciadas en los trabajos de Chenery (1982) y Syrquin (1988).

Una metodología alternativa para analizar los cambios en la estructura productiva, es realizar el estudio desde la perspectiva de la demanda, la cual permite explicar el crecimiento del producto por la influencia que ejercen la demanda doméstica, y el comercio exterior sobre el aparato productivo. Ésta es una aproximación Keynesiana, que permite considerar de manera implícita la influencia de la demanda en el nivel de producción.

En este apartado se analiza, si el comportamiento del sector manufacturero, en las últimas cuatro décadas, estuvo ligado a un cambio en las fuentes del crecimiento industrial. Para ello, se estima la contribución del incremento de la demanda interna (nacional), del proceso de sustitución de importaciones (manufacturas) y de la expansión de las exportaciones (manufacturas) al crecimiento del producto manufacturero.

La metodología tradicional formulada por Chenery y Syrquin (1989), la cual analiza los determinantes de la dinámica del crecimiento industrial, a través, de las fuentes de crecimiento de la demanda ${ }^{2}$, se emplea para la identificación de los factores que lideran el comportamiento del sector manufacturero. La metodología permite descomponer el crecimiento del producto industrial en tres factores ${ }^{3}$ : el crecimiento de la demanda interna, el incremento de las exportaciones (demanda externa) y la sustitución de importaciones. La descomposición de Chenery y Syrquin (1989) permite recoger, en cierta medida, los cambios estructurales sobre la industria manufacturera, a partir de la implementación de las políticas de sustitución de importaciones, década de los setenta, y de políticas de apertura económica, década de los noventa. Por lo tanto, se espera que si el proceso de liberalización comercial ha tenido éxito, tuviese un impacto positivo en el crecimiento explicado por un incremento en las exportaciones, acompañado de un impacto negativo originado de la sustitución de importaciones debido a la apertura de los noventa.

El análisis de fuentes de crecimiento del sector manufacturero

2 Desde luego hay que aclarar que este enfoque es criticable, ya que se descuidan los factores de oferta, tal como, el incremento en la productividad (debido al progreso tecnológico), que inciden en la demanda a través de los precios. De igual manera es criticable el tratamiento de la sustitución de importaciones como un factor de demanda, ya que puede argüirse como la oferta externa.

3 Las técnicas de descomposición son una herramienta importante para aclarar las fuentes del crecimiento temporal de algunas variables. El análisis de descomposición estructural se define como un método que permite identificar las principales transformaciones en una economía mediante cambios de estática en grupos claves de parámetros (Maldonado, 2010). La metodología de análisis de descomposición estructural tiene similitudes con la medición del crecimiento económico, en donde se busca descomponer la tasa de crecimiento de la producción agregada entre las contribuciones del crecimiento de cada uno de sus factores productivos. 
se basa en Chica (1982:80-81). La Ecuación 1 parte de la identidad de oferta y demanda doméstica , donde $\overline{\mathrm{I}} \Delta \mathbf{D}$ indica la contribución de la demanda doméstica ${ }^{4}$ al crecimiento del producto industrial $(\Delta \mathrm{P})$, $\overline{\mathrm{D}} \Delta \mathbf{i}$ la sustitución de importaciones, y $\Delta \mathbf{X}$ la contribución de las exportaciones.

$$
\Delta \mathrm{P}=\mathrm{T} \Delta \mathrm{D}+\overline{\mathrm{D}} \Delta \mathrm{i}+\Delta \mathrm{X}
$$

(Ecuación 1)

La Tabla 1 presenta las fuentes de crecimiento del producto industrial manufacturero colombiano según sus fuentes de demanda. El crecimiento de la producción bruta industrial, es clasificada en tres fuentes de crecimiento: una derivada de la demanda doméstica (T $\Delta \mathrm{D})$, otra surge de la sustitución de producción importada $(\overline{\mathbf{D}} \Delta \mathbf{i})^{5}$ y una tercera se relaciona con incremento de la demanda externa (exportaciones) $(\Delta \mathbf{X})$.

Los cálculos indican que la demanda interna lidera la dinámica del sector manufacturero. Por ello, el crecimiento del producto industrial manufacturero en el largo plazo, se encuentra sustentado por el desempeño de la demanda doméstica a pesar de su comportamiento cíclico e irregular, siempre ha contribuido en mayor proporción al crecimiento industrial, en comparación con las exportaciones o el componente de sustitución de importaciones.

\section{Tabla 1}

Fuentes de crecimiento del producto industrial manufacturero colombiano (Período 1970-2008)

\begin{tabular}{cccc}
\hline Períodos & $\begin{array}{c}\text { Contribución de la } \\
\text { demanda doméstica } \\
\text { al PIB industrial }\end{array}$ & $\begin{array}{c}\text { Contribución de la sustitución } \\
\text { de importaciones al PIB } \\
\text { industrial }\end{array}$ & $\begin{array}{c}\text { Contribución de las } \\
\text { exportaciones al PIB } \\
\text { industrial }\end{array}$ \\
\hline $1970-1974$ & $74,22 \%$ & $-6,66 \%$ & $32,44 \%$ \\
$1975-1979$ & $62,11 \%$ & $-2,93 \%$ & $40,82 \%$ \\
$1980-1984$ & $100,48 \%$ & $-4,11 \%$ & $3,63 \%$ \\
$1985-1989$ & $73,66 \%$ & $-1,01 \%$ & $27,35 \%$ \\
$1990-1994$ & $188,12 \%$ & $-177,13 \%$ & $89,01 \%$ \\
$1995-1999$ & $62,31 \%$ & $-10,26 \%$ & $47,95 \%$ \\
$2000-2004$ & $33,48 \%$ & $-4,39 \%$ & $70,91 \%$ \\
$2005-2008$ & $105,21 \%$ & $-27,42 \%$ & $72,22 \%$ \\
\hline
\end{tabular}

Fuente: Elaboración propia, a partir de la base en datos DANE-EAM

4 La demanda doméstica se calcula a partir de la siguiente ecuación: $D=P+M-X$, donde $D$ representa la demanda doméstica de bienes industriales, $\mathrm{P}$ la producción doméstica de bienes industriales, $\mathrm{M}$ las importaciones de bienes manufactureros y $\mathrm{X}$ las exportaciones de bienes manufactureros. Para los cambios entre los periodos 1 y 2 se sigue la siguiente ecuación $\Delta \mathrm{P}=\mathrm{i} 1$ $\left(D_{2}-D 1\right)+D_{2}\left(i_{2}-i 1\right)+\Delta X$, donde $i$ es el coeficiente de sustitución de importaciones $(\mathrm{i}=[\mathrm{P}-\mathrm{M}] /[\mathrm{P}$ $+\mathrm{M}-\mathrm{X}]=[\mathrm{P}-\mathrm{M}] / \mathrm{D})$. Para un análisis más detallado del desarrollo matemático de las fuentes del crecimiento manufacturero consulte Chica (1982).

5 Sustitución de importaciones, proceso mediante el cual la reducción de las importaciones implica una redirección de la demanda hacia producción doméstica. 
La incidencia de la demanda externa en el crecimiento del producto ha venido aumentando su participación de manera sustancial durante todo el periodo analizado. Indica que la dinámica del sector exportador ha venido mejorando, y esto se muestra en la mayor participación como fuente del crecimiento del sector manufacturero a partir de la década de los noventa, ya que durante los setenta y ochenta su desempeño fue marginal. Por su parte, la participación de la sustitución de importaciones en el crecimiento del producto manufacturero ha mostrado coeficientes negativos en todos los periodos bajo estudio; sin embargo, este efecto ha sido menor, con excepción de inicios de la década de los noventa, lo cual implica un avance del país hacia un proceso de "apertura hacia adentro"6.

Con respecto a las exportaciones, se resalta que a lo largo del periodo de post apertura se observan dos comportamientos característicos. Durante el periodo 19901994, la contribución de la demanda interna al crecimiento del PIB manufacturero fue el más significativo; el aporte de las exportaciones fue mínimo, en contraste con la gran contracción del componente de sustitución de importaciones. Por este motivo, la demanda local por bienes industriales de consumo e inversión en este periodo, debió ser mayor para compensar la baja contribución de las exportaciones y la contribución negativa de la sustitución de importaciones. En el periodo 1995-1999, la participación de la demanda interna es el componente que más contribuye al crecimiento del producto industrial, aunque las exportaciones tienen una participación relativamente superior al primer quinquenio de la década de los noventa, el proceso de sustitución de importaciones siguió siendo negativo.
Distinto es el panorama que se presenta a lo largo de la primera mitad de la década del dos mil. Se presenta una reducción importante y relativa del efecto negativo sobre el crecimiento de la producción industrial de la sustitución de importaciones, para el caso de las exportaciones es notable su participación en el crecimiento del PIB industrial, tanto así, que poseen la mayor participación, superior a la contribución de la demanda interna, para el primer quinquenio del 2000. Sin embargo, para finales del periodo bajo estudio, la demanda interna vuelve a tener mayor participación dentro del producto manufacturero.

La década de los noventa marca la entrada de Colombia en el grupo de países emergentes que emprendieron reformas económicas, combinación de apertura comercial y reforma del Estado, principalmente a través de privatización y reducción del aparato estatal. La apertura económica es sencilla, casi simple. La apertura expone a las empresas domésticas a la competencia internacional, y las diferencias de productividad dificultan la penetración competitiva de sus productos en el exterior, obligadas a vender en el mercado interno, teniendo que aumentar la productividad para reducir precios frente a la competencia externa.

Por su parte, a finales de la década de los noventa y mediados de la década del dos mil, se constatan mejoras en el desempeño productivo de las empresas. Como indica Malaver (2007:321), estas mejoras se inscribieron en estrategias defensivas de racionalización de costos, y aunque hubo un breve periodo en que se acompañaron de un importante proceso de modernización técnica, primó la reducción de costos para elevar la eficiencia. Ante la

6 Se refiere al proceso de liberalización comercial, caracterizado por el mayor dinamismo de las importaciones, causante de amplios déficits comerciales. 
caída de la demanda interna las estrategias de racionalización de las empresas del sector manufacturero, se combinaron con estrategias exitosas, a primera vista, penetración y ampliación de sus mercados externos.

\section{Cambio estructural en la industria manufacturera colombiana $^{7}$}

El cambio estructural es prácticamente una de las características más relacionadas con el crecimiento económico. Sólo en casos particulares, el crecimiento económico podría concebirse como un proceso de expansión uniforme y de convergencia a tasas similares en todos los sectores productivos, ya que no todos tienen el mismo poder de inducir aumentos de productividad, de promover la expansión de otros sectores o de beneficiarse de tasas altas de crecimiento de las demandas internas y externa, o de generar empleos de alta productividad (Vera, 2000).

Quienes destacan la relación inseparable entre el crecimiento económico y el cambio estructural, afirman que el cambio estructural es cierto tenor para generar condiciones de crecimiento sostenido y desarrollo económico. Como indica Chenery y Syrquin (1989:16), éste puede retardar o estancar el crecimiento si en sí mismo muestra un lento ritmo o si los cambios se enfocan hacia sectores con bajo dinamismo. En la mayoría de las economías desarrolladas y en vía de desarrollo, el tránsito de estructuras productivas de baja complejidad relacionadas con la explotación de bienes primarios y recursos naturales hacia estructuras más complejas ligadas al fenómeno de la industrialización son una evidencia empírica muy importante, que para los economistas clásicos del desarrollo, es la principal vía para la sustentación de procesos de desarrollo económico en el largo plazo (Vera, 2009:91).

A continuación se explica la medida de cambio estructural que será utilizada en esta investigación. Primero, cuando se habla de estructura se hace referencia a la estructura productiva del sector manufacturero. Segundo, para la medición del cambio estructural se pueden utilizar varias metodologías, sin embargo, por su sencillez y fácil interpretación fue seleccionado el índice propuesto por Schiavo-Campo (1978:261-263), que consiste en la sumatoria de los valores absolutos de los cambios en la participación (sobre el producto total) de cada sector de la economía, dividido entre dos. Tercero, es preciso definir una unidad de tiempo, que recoja la recomposición de la estructura productiva (con ello se busca minimizar los efectos asociados a los cambios cíclicos) y permita comparar la velocidad de los cambios.

El Índice de Cambio Estructural (ICE) de Schiavo-Campo viene dado por la Ecuación 2.

$$
I C E=\frac{\sum_{i=1}^{n}\left|p_{i t}-p_{i(t+k)}\right|}{2} \quad \text { (Ecuación 2) }
$$

donde $\mathrm{i}=$ rama o sector productivo

pi $=$ participación del producto

del sector i en el producto total

$(\mathrm{t}+\mathrm{k})=$ periodo o unidad de tiempo

La Ecuación 2 busca determinar los cambios en el tiempo en la partición de cada uno de los sectores económicos empleados en el cálculo del ICE, la

7 Algunas ideas planteadas en esta sección fueron tomadas de Vera (2009). 
sumatoria en el denominador se ha dividido entre dos pare evitar que el índice sea afectado por la doble contabilidad de los cambios en las participaciones de cada uno de los sectores estudiados.

A los efectos del cálculo para la economía colombiana, se ha tomado la clasificación sectorial del PIB que provienen de las cuentas nacionales publicadas por el Departamento Administrativo Nacional de Estadísticas (a un dígito) en el año 2012. Excluyendo los impuestos indirectos, la estructura corresponde a nueve sectores productivos, agricultura, explotación de minas y canteras, industria manufacturera, electricidad, gas y agua, comercio, restaurante y hoteles, transporte almacenamiento y comunicaciones, establecimientos financieros, seguros, inmuebles y servicios a las empresas, y servicios comunales, sociales y personales. El PIB para cada uno de estos sectores se ha tomado a precios corrientes $^{8}$ para refinar la precisión en el cálculo de la participación pi. El periodo de cálculo del Índice de Cambio Estructural (ICE) es 1970-2005.

El índice de cambio estructural se ha calculado para dos periodos diferentes (o unidad de tiempo) para periodos de diez años y de cinco años. La Tabla 2 muestra la evolución del índice para cada uno de los periodos, así como el crecimiento promedio del producto real total ${ }^{9}$.

Tabla 2

\section{Índice de cambio estructural en Colombia}

\begin{tabular}{ccc}
\hline Años & $\begin{array}{c}\text { Índice de Cambio Estructural } \\
\text { ICE }\left(\sum \mid \mathbf{p}_{\text {it }}-\mathbf{p}_{\mathbf{i}}(\mathbf{t}+\mathbf{k}) \mathbf{l}\right)(\%)\end{array}$ & $\begin{array}{c}\text { Tasa del crecimiento } \\
\text { del PIB (\%) }\end{array}$ \\
\hline Período: 5 años & 3,8 & 6,5 \\
\hline $1970-1974$ & 3,1 & 5,0 \\
$1975-1979$ & 4,8 & 2,4 \\
$1980-1984$ & 4,6 & 4,4 \\
$1985-1989$ & 9,5 & 4,1 \\
$1990-1994$ & 7,8 & 1,4 \\
$1995-1999$ & 4,4 & 3,1 \\
$2000-2005$ & & 5,7 \\
\hline Perído: 10 años & 4,0 & 3,4 \\
$1970-1979$ & 8,9 & 2,8 \\
$1980-1989$ & 12,6 & 2,5 \\
\hline $1990-1999$ & 9,1 & \\
$2000-2008$ & & \\
\hline
\end{tabular}

Fuente: Elaboración propia, a partir de las cuentas nacionales DANE

Con el objetivo de que los resultados no presenten sesgo, debido al tratamiento estadístico de los datos.

$9 \quad$ Los problemas de divisibilidad al terminar con un periodo de seis años para la data quinquenal. 
En esta investigación, se analiza la evolución del índice de Schiavo-Campo, evidenciando en las últimas décadas, el acelerado proceso de cambio estructural en la estructura productiva de la economía. La evidencia es consistente para distintos periodos (décadas y quinquenios).

El índice de cambio estructural muestra que los cambios en la partición de cada uno de los sectores productivos en la producción agregada es más acentuado en año recientes que en años precedentes. La evidencia empírica muestra que existe una relación inversa entre la tasa promedio de crecimiento del PIB y el ICE, una contracción del producto agregado va acompañado de una variación positiva en la medida de cambio estructural. Por ejemplo, observando en el primer quinquenio de la década de los ochenta, un periodo de estancamiento y durante el cual el crecimiento del producto real fue de $2,4 \%$ el índice de cambio estructural se incrementó en un $56,13 \%$ (con respecto al quinquenio de 1975-1979).

Una nueva aceleración en el índice vuelve a presentarse en el primer quinquenio de la década de los noventa, donde la tasa de crecimiento no es tan bajo, sin embargo, este comportamiento del índice coincide con el proceso de apertura económica que se implementó a inicio de la década, en este periodo el índice alcanza su mayor nivel $(9,5 \%)$ y presenta una tasa de crecimiento del 106,32\% (con respecto al quinquenio 1985-1989). Este patrón también se puede observar en los periodos de diez años, donde los valores tomados por el índice son más elevados, desde luego esto es consistente, si se tiene en cuenta las transformaciones de largo plazo en los sectores productivos, éstas son más profundas en comparación con periodos de tiempo más cortos.

Desde mediados de los años setenta en Colombia se llevó a cabo un cambio estructural del modelo de desarrollo basado en la sustitución de importaciones y en la protección a la industria nacional, por uno sustentado en la liberalización de los mercados y la competencia externa. Este cambio se adoptó con la idea de mejorar las condiciones de competitividad y productividad de la industria nacional. Con la ayuda del Índice de Cambio Estructural se ha encontrado que la profundización de esta reforma a partir de la década de 1990 ha disminuido el crecimiento del sector industrial y dificultó aún más la transformación y diversificación de la industria.

La estructura productiva nacional, muestra una mayor dinámica de crecimiento del sector terciario, en relación con el comportamiento del sector industrial. Sin embargo, a diferencia de los países desarrollados ${ }^{10}$ donde el proceso de tercerización responde a un cambio estructural impulsado por las ganancias permanentes de productividad, el cambio técnico, la transformación productiva industrial, y al desarrollo de servicios modernos altamente cualificados basados en el conocimiento, la ciencia y la tecnología, con importantes eslabonamientos con la actividad manufacturera; en el caso colombiano, se presenta como consecuencia del progresivo deterioro y pérdida de competitividad del sector agrícola y manufacturero.

10 Contrario a la acontecido en otros países latinoamericanos y en muchas de las economías asiáticas en los que la industria se vio fortalecida antes de entrar en una fase de tercerización de la economía, en Colombia está sucediendo todo lo opuesto, con el imperativo de que buena parte del sector de los servicios es aún de carácter informal (Garay, 1998). 


\section{Apertura económica y desindustrialización en Colombia}

Durante los años 1975-2012, Colombia experimentó un proceso de desindustrialización a través del cual la participación del sector industrial en la producción agregada descendió de un $24 \%$ hace tres décadas a un $15 \%$ hace una década y se perfila hacia tan sólo un $9 \%$ $12 \%$ para el periodo 2012-2020 (Clavijo et al, 2012:11). Durante el periodo post aperturista ha disminuido el crecimiento y la participación del sector manufacturero en el agregado económico, así como la inserción de los bienes manufactureros en el sector externo. El efecto de la apertura comercial fue el deterioro estructural del crecimiento de la producción de los bienes transables y no transables y el permanente déficit comercial, que se caracterizó por el incremento rápido y excesivo de las importaciones en respuesta, a la eliminación de las restricciones cuantitativas, la reducción arancelaria y a la apreciación del tipo de cambio.

La evidencia presentada en esta sección demuestra que en las últimas tres décadas la economía colombiana ha venido transitando por un acelerado proceso de cambio en sus estructura productiva con un sesgo desindustrializador.

\subsection{Las importaciones industriales y sus efectos sobre el valor agregado industrial en Colombia}

En este parte se utiliza la modelación econométrica para dar mayor compresión al proceso de cambio estructural en la industria manufacturera durante el periodo el periodo de apertura económica, y contrastar los resultados obtenidos en secciones anteriores. Entre los principales resultados se tienen: aumento de las importaciones, revaluación del peso debido a la liberalización cambiaria, mayor com-petencia a la industria nacional como resultado de la apertura a través del desmonte arancelario y la liberalización de los mercados de capitales. Los anteriores resultados tuvieron efectos adversos sobre la industria manufacturera nacional.

Por otra parte, García (2002:195196):

"No hay que olvidar que el lenguaje básico que determina el comportamiento del mercado es el que define los precios. Considerando este postulado, cuando se producen cambios en el tipo de cambio real, ellos se manifiestan como en efecto masivo que alteran los precios relativos $y$ la relación de intercambio de los bienes y servicios de producción nacional frente al resto del mundo. De este modo, cuando el tipo de cambio se revalúa, no como respuesta al crecimiento de la producción y las exportaciones netas, sino como consecuencia de los flujos financieros y de capital, el resultado es una distorsión monetaria de los precios y los términos de intercambio exteriores. Esta distorsión eleva el precio relativo de las exportaciones frente a los mercados externos y disminuye el precio relativo de las importaciones en el mercado doméstico, tal como ha ocurrido en el mercado colombiano durante el periodo de la apertura".

Lo anterior indica que el proceso de revaluación del tipo de cambio favorecido por el proceso de apertura, incidió en el aumento de las importaciones en detrimento de las exportaciones domésticas, generando pérdida de competitividad de la producción interna. Esto tiende a disminuir en el largo plazo la producción de las ramas agrícolas e industriales de la economía.

\subsection{Modelo econométrico}

La implementación de modelos de datos panel permite mayor flexibilidad 
para diferenciar comportamientos entre observaciones, es decir, permite modelar la heterogeneidad entre los diferentes subsectores que hacen parte del sector manufacturero. Además, permite clasificar los efectos económicos que no pueden distinguirse sólo con el uso de datos de corte transversal o series de tiempo.

Esta metodología permite obtener modelos con o sin ponderaciones entre los miembros de los datos de panel. Un modelo con ponderaciones asume que no existe una tendencia uniforme entre los sectores industriales frente al crecimiento del valor agregado industrial. La estimación sin ponderación asume igual peso entre las variables de sección cruzada, esto es, que los sectores por tamaño de empresa tienen el mismo efecto en el crecimiento industrial colombiano.

Se utiliza la metodología de datos panel, estimándose un modelo de datos panel balanceado, por cuanto posibilita solucionar problemas de endogenidad resultantes de variables omitidas periodo a periodo, al igual que en la unión de cortes transversales, permite medir efectos no observables en la muestra de corte transversal, y mejoran la precisión de las estimaciones (Baltagi, 2005:4).

En este estudio, sobre política industrial, los efectos individuales vienen siendo los subsectores industriales ${ }^{11}$. Por tanto, los efectos individuales son invariables en el tiempo, y afectan de manera directa las decisiones que se tomen sobre la política industrial, tales como la legislación tributaria, estímulos por parte del sector gubernamental a las empresas del sector en función de su tamaño.

Para medir el crecimiento de la industria se utiliza el valor agregado por subsector industrial (CIIU Revisión de 2 a 3 dígitos) y a través de las importaciones por subsectores industriales se miden los resultados de políticas implementadas por la liberalización económica. Adicionalmente se utiliza dummy para determinar si existió un cambio estructural en el crecimiento industrial después de la apertura. También fue incluida la variable exportaciones industriales como variable de control ${ }^{12}$.

La Ecuación 3 muestra el modelo de datos panel a estimar, también, incluye las variables independientes y la variable dependiente.

$\ln V_{a t i}=\alpha+\beta \ln M_{\mathrm{ti}}+\beta \ln X_{\mathrm{ti}}+\beta \operatorname{lnE}+\theta_{0}$ Apertura $+\theta_{1} \mathrm{AM}_{\mathrm{ti}}+$
$\theta_{2} \mathrm{AX}_{\mathrm{ti}}+a_{\mathrm{ti}}+\mu_{\mathrm{it}}$
$\quad$ (Ecuación 3)

Siendo la variable dependiente $V_{a}$ el valor agregado por subsectores industriales en el periodo $t$ y subsectorindustrial $i$. Las siguientes variables conforman el conjunto de variables explicativas del modelo a estimar descrito en la Ecuación 3: M las importaciones por subsectores industriales

11 Estos subsectores industriales fueron tomados de la Encuesta Anual Manufacturera (EAM), según la Código Internacional Industrial Uniforme (CIIU) Revisión a 2 dígitos. Hasta el año 2000, los datos corresponden a la base CIIU Revisión a 2 dígitos. A partir del año 2001, la información fue recopilada de la base CIIU Revisión a 3 dígitos. Para efectos de este trabajo, fue empalmada la base de datos con las tablas de equivalencia que presenta el DANE. La base de datos quedó conformada por 18 subsectores con datos expresados en su totalidad a partir de la base CIIU Revisión a 2 dígitos.

12 Se corrió otro modelo incluyendo otras variables disponibles de la base de datos como el número de establecimientos industriales, número de personas empleadas en la industria manufacturera; y los parámetros de las variables objeto de estudio no sufrieron cambios significativos, incluso los resultados se hacen más consistentes con lo planteado en la hipótesis, lo que demuestra que no hay inconvenientes de variables omitidas. 
en el periodo $t$ y subsector industrial $i, X$ las exportaciones por subsectores industriales en el periodo $t$ y subsector industrial $i$, $E$ el número de establecimientos por subsectores industriales en el periodo $t \mathrm{y}$ subsector industrial $i, \theta_{0}$ Apertura, la dummy que permite hacer un test de cambio estructural en el modelo cuyo objetivo es evaluar la hipótesis del estructural con un sesgo desindustrializador (pérdida de valor agregado) por cuenta de la reforma liberal agudizada en la década del 90.

La variable dummy es binaria, y trata de medir un factor cualitativo más no cuantitativo, por tanto es igual a $1 \mathrm{si}$ la muestra de las variables son tomadas del periodo de apertura (1992-2008) y es igual a 0 si la muestra no es en el periodo de apertura (1970-1991). Si esta variable es significativa, entonces prueba que la apertura generó un cambio estructural sobre el valor agregado industrial, es decir, el valor agregado se afectó de forma positiva o negativa (esto depende del signo arrojado por el coeficiente de la dummy) a partir de la apertura.

Cuando la variable dummy se combina con la variable importaciones, se crea otra variable denominada AM y permite determinar cuánto disminuyó (o aumentó) el crecimiento del valor agregado por el aumento de las importaciones (M) como consecuencia de la apertura, similar interpretación se puede realizar para la variable exportaicion. En el modelo se toman las tasas de crecimiento de las variables (logaritmo) y no sus niveles. La técnica de estimación econométrica usada es la de datos de panel, tanto por Efectos Fijos (EF) como por Efectos Aleatorios (EA), la cual permite darle un mejor uso a la información, a diferencia de técnicas como la de corte transversal, puesto tiene en cuenta y valora los cambios que se dan en el valor agregado, las importaciones y las exportaciones industriales de acuerdo con cada subsector industrial a través tiempo.

\subsubsection{Resultados de la estimación econométrica}

Los resultados de este modelo fueron consistentes con lo esperado usando el métodos de datos de panel: efectos aleatorios, indicando que las variables inobservables ai (políticas salariales y de contracción, adopción de patentes, plan de inversiones, entre otras) son poco importantes en términos relativos (esto se debe a la presencia de una varianza pequeña en relación con la varianza $\mu(\sigma \mu)$. A continuación se presenta la salida del método de estimación de efectos aleatorios ${ }^{13}$ (Tabla 3 ).

13 Se llevó a cabo la prueba de Hausman para determinar la mejor estimación entre el modelo de efectos fijos y el de efectos aleatorios, el resultado de esta prueba arrojo que se debe rechazar la hipótesis nula de ortogonalidad (no correlación entre los regresores y el término de error, es decir, que $\mathrm{E}\left[\varepsilon \_\right.$it IX_it=0] al $5 \%$ de significancia, por lo tanto el estimador de efectos aleatorios es la mejor opción para estimar el panel. La estimación del modelo de efectos aleatorios presento problemas de autocorrección, ya que los errores de diferentes unidades de corte transversal estaban correlacionados (correlación contemporánea), adicionalmente los errores también se encontraban correlacionados dentro de cada unidad de corte transversal (correlación serial). Para la detección de la autocorrelación en el modelo de efectos aleatorios se realizó la prueba de Breusch y Pagan para identificar problemas de correlación contemporánea en los residuos. El problema de autocorrelación fue resuelto mediante un la inclusión de un ajuste autoregresivo de primer orden $\mathrm{AR}(1)$, con lo anterior se remueve en el modelo de regresión de sección cruzada en el tiempo la autocorrelación de los disturbios a través del tiempo. Se realizaron pruebas para la detección de multicolinealidad y heteroscedasticidad, sin embargo, no existió evidencia estadística en el modelo estimado de efectos aleatorios. 
Se usaron en total 702 observaciones provenientes de los 18 subsectores manufactureros, identificados en la Encuesta Anual Manufacturera (EAM) del DANE, distribuidos a lo largo de los 39 años que cubre el periodo bajo estudio, el modelo de datos panel descrito por la Ecuación 3 en su conjunto es estadísticamente significativo por el método de efectos aleatorios, y el coeficiente de determinación (R2) entre la variable dependiente y las variables independientes es bueno, ya que cerca de un $54 \%$ del comportamiento del valor agregado industrial está explicado por las variables independientes incluidas en el modelo.

\section{Tabla 3}

\begin{tabular}{|c|c|c|c|c|c|c|c|}
\hline \multicolumn{2}{|c|}{ Valor Agregado } & Coeficiente & $\begin{array}{l}\text { Desviación } \\
\text { estándar }\end{array}$ & $\mathbf{z}$ & $p>z$ & [95\% Conf. & Interval.] \\
\hline \multicolumn{2}{|l|}{ Importaciones } & 0,0181346 & 0,076244 & 2,38 & 0,017 & 0,003191 & 0,0330781 \\
\hline \multicolumn{2}{|l|}{ Exportaciones } & 0,0301786 & 0,0443543 & 5,21 & 0,000 & 0,14422753 & 0,3181409 \\
\hline \multicolumn{2}{|l|}{ Establecimientos } & 0,2312081 & 0,0056229 & 5,37 & 0,000 & 0,019158 & 0,0411992 \\
\hline \multicolumn{2}{|l|}{ Apertura } & $-0,090723$ & 0,069898 & $-3,36$ & 0,001 & $-0,1436221$ & $-0,037824$ \\
\hline \multicolumn{2}{|c|}{ Apertura_Importaciones } & $2,24 \mathrm{e}-08$ & $6,61 \mathrm{e}-09$ & 3,38 & 0,001 & $9,41 \mathrm{e}-09$ & $3,53 e-08$ \\
\hline \multicolumn{2}{|c|}{ Apertura_Exportaciones } & $7,74 \mathrm{e}-08$ & $1,77 e-08$ & 4,37 & 0,000 & $4,26 e-08$ & $1,12 \mathrm{e}-07$ \\
\hline \multicolumn{2}{|l|}{ Constante } & 12,0098 & 0,2848154 & 42,17 & 0,000 & 11,45157 & 12,56803 \\
\hline rho_ar & \multicolumn{7}{|c|}{$0,94472615 \quad$ (estimated autocorrelation coefficient) } \\
\hline Sigma_u & \multicolumn{7}{|c|}{0,50192692} \\
\hline Sigma_e & \multicolumn{7}{|c|}{0,0856162} \\
\hline rho_fow & \multicolumn{7}{|c|}{$0,96287186 \quad$ (fraction of variance due to $u \_i$ ) } \\
\hline Theta & \multicolumn{7}{|c|}{0,61647599} \\
\hline $\begin{array}{l}N^{0} \text { de observa- } \\
\text { ciones }\end{array}$ & \multicolumn{2}{|l|}{702} & \multicolumn{3}{|c|}{ Variable dependiente } & \multicolumn{2}{|c|}{ Valor Agregado } \\
\hline Variable grupo & \multicolumn{2}{|l|}{18} & \multicolumn{3}{|l|}{$N^{\circ}$ de grupos } & \multicolumn{2}{|l|}{18} \\
\hline R-sq: Within & \multicolumn{2}{|c|}{0,5910} & \multicolumn{3}{|c|}{ Observaciones por grupo } & \multicolumn{2}{|l|}{39} \\
\hline Between & \multicolumn{2}{|c|}{0,5311} & \multicolumn{3}{|l|}{ Promedio } & \multicolumn{2}{|l|}{39} \\
\hline Overall & \multicolumn{2}{|c|}{0,5372} & \multicolumn{3}{|l|}{ Máximo } & \multicolumn{2}{|l|}{31} \\
\hline Random effects & \multicolumn{2}{|c|}{ u_i Gaussian } & \multicolumn{3}{|c|}{ Wald chi2(6)= } & \multicolumn{2}{|l|}{995,63} \\
\hline $\operatorname{Corr}\left(\mu_{i}, X b\right)$ & \multicolumn{2}{|c|}{0 (assumed) } & \multicolumn{3}{|l|}{ Prob $>$ chi2 $=$} & \multicolumn{2}{|l|}{0,0000} \\
\hline
\end{tabular}

Fuente: Elaboración propia 
Por otra parte, las variables independientes son significativas a un nivel de significancia de un $5 \%$ y esto es realmente importante para el caso de la variable dummy Apertura, pues se comprueba que la implementación de la política de liberalización causó un cambio estructural negativo (el signo del parámetro es negativo) en el crecimiento de la industria medido a través del valor agregado. Los resultados del modelo estimado se presentan en la Ecuación 4.

$\ln \mathrm{Vati}_{\mathrm{i}}=12,098+0,018 \ln \mathrm{M}_{\mathrm{ti}}+0,030 \ln \mathrm{X}_{\mathrm{ti}}+0,312 \ln \mathrm{E}-$

0,091Apertura $+2,24 \mathrm{e}-08 \mathrm{AM}_{\mathrm{ti}}+7,74 \mathrm{e}-08 \mathrm{AX} \mathrm{ti}_{\mathrm{t}}$

(Ecuación 4)

De forma concreta, la tasa de crecimiento del valor agregado industrial es en promedio después de la apertura 2,2\% más baja en comparación con el periodo antes de la apertura. Lo cual se acerca mucho a la realidad del crecimiento de la industria manufacturera, pues durante el periodo 1970-1989 (pre-apertura) el valor agregado industrial creció $4,72 \%$, mientras que en el periodo 1990-2008 (post-apertura) creció al 2,4\%.

El efecto de las importaciones en el valor agregado antes y después de la apertura no es el mismo. Antes de la apertura un crecimiento de un $1 \%$ en las importaciones producía un incremento positivo del valor agregado de 0,018\%, que se debió a la importación de bienes intermedios y de capital para la industria, mostrando la dependencia de las importaciones de bienes con alto valor agregado.

Después de la apertura económica, el efecto de las importaciones, medido a través de la variable combinada dummy Apertura con la variable Importaciones es positivo, es decir, un crecimiento inusual de las importaciones como consecuencia de la apertura afectó levemente el crecimiento del valor agregado, lo cual indica una mayor dependencia de la industria manufacturera de las importaciones, tal como se expuso anteriormente.

En el caso de la apertura, las importaciones no están generando un gran efecto en el valor agregado, lo cual no es coherente con los niveles de importaciones reportados por la industria manufacturera después de la apertura. Se podrían clasificar por una parte las importaciones que actúan como complemento a la producción industrial y son las generadoras de efectos positivos al crecimiento del valor agregado, y por otro lado las importaciones que compiten con la producción doméstica y generan un estancamiento en el crecimiento del valor agregado industrial. Esta última podría ser la característica más visible después de la apertura, tal como indica la variable Apertura.

La evidencia estadística indica que si bien existe una relación positiva entre la tasa de crecimiento del valor agregado industrial y las importaciones, también se muestra una relación negativa entre el monto de importaciones y el valor agregado industrial.

Por su parte el coeficiente de la variable exportaciones es positivo, tal como se esperaba, y al combinarse con la dummy Apertura el efecto positivo sobre el valor agregado es levemente mayor, es decir, que el efecto exportación sobre el valor agregado no es lo suficientemente fuerte para atenuar el efecto negativo de la apertura, medido a través de la dummy Apertura. Por lo tanto el aumento de las exportaciones de ciertas ramas de manufacturas básicas, especialmente después de la crisis de finales de la década de los noventa, no fueron suficientes para contrarrestar la caída del valor agregado como consecuencia del crecimiento de las importaciones industriales. 
El coeficiente de la variable establecimientos es positivo; este signo era el esperado. Presenta un notable efecto sobre el valor agregado, ya que un incremento en un $1 \%$ en el número de establecimientos pertenecientes al sector manufacturero genera un aumento de un $0,23 \%$ en el crecimiento del valor agregado industrial. Desde luego esto es consistente, ya que mayor número de establecimientos industriales generarían un incremento en el producto industrial y en la creación de valor.

\section{Conclusiones}

El crecimiento industrial en Colombia en las últimas cuatro décadas, ha estado impulsado principalmente por el comportamiento de la demanda doméstica, esto refleja la vulnerabilidad del patrón de crecimiento industrial a las fluctuaciones de la demanda interna. Entre tanto, el sector exportador no ha sido un factor determinante del crecimiento económico en Colombia, a diferencia del propósito que se le asignó en el modelo de apertura. El débil desempeño del sector exportador hace inviable la sostenibilidad del modelo de internacionalización al afianzar la tendencia estructural de la apertura hacia adentro en lugar de una apertura hacia afuera.

La evidencia presentada en este estudio indica que la economía colombiana ha venido exponiendo en los últimos tiempos un proceso acelerado de cambio en la estructura de la producción con un sesgo desindustrializador. Este proceso de transformación productiva puede ser crucial para entender hacia dónde está apuntando el patrón de especialización de la economía colombiana en el largo plazo, y qué perspectivas se tienen para consolidar en el futuro un crecimiento robusto $y$ sostenible.

Por su parte, la prueba econométrica y la construcción del Índice de Cambio Estructural demostraron la existencia de un cambio estructural de efecto negativo en el crecimiento del valor agregado industrial después de 1992, periodo de profundización de la política aperturista. Además, el crecimiento de las importaciones industriales en el periodo de liberalización tuvo un efecto negativo en el crecimiento de la industria nacional.

Es importante para el país avanzar en la definición y consolidación de una estrategia de desarrollo de largo plazo apalancada en un nuevo paradigma sectorial, de innovación y desarrollo de capacidades tecnológicas que le permitan modificar progresivamente el patrón de desarrollo de la estructura productiva vigente, en favor de actividades generadoras de valor agregado, la difusión de conocimientos e innovación. La expansión de capacidades en sectores manufactureros de intensidad tecnológica le permitirá al país ampliar y modificar sus modalidades tradicionales de inserción en la economía mundial. Es necesario, así mismo, ampliar y profundizar los esquemas y mecanismos de integración regional existentes para favorecer los procesos de diversificación de las exportaciones manufactureras.

La experiencia reciente de las dos últimas décadas muestra que sistemáticamente se busca promover el desarrollo e impulsar el crecimiento económico por la vía exclusiva de la liberalización comercial, sobre la base del crecimiento y diversificación del sector exportador y se persiste de manera obstinada en la adopción de una política industrial neutral, donde el mercado externo determina los sectores industriales 
capaces de competir en los mercados internacionales.

Aunque el proceso de desarrollo exige cambios en las formas de producir y en la estructura de la economía, estos no pueden darse sin que la transformación abra paso a las fuerzas del progreso técnico. El problema de la economía colombiana parece ir justamente en dirección opuesta.

Colombia generar políticas industriales de largo plazo, donde el cambio tecnológico y la inserción externa de los bienes manufacturados con alto valor agregado sean los ejes centrales y articuladores, esta política industrial debe ser ambiciosa y prudente. Se debe concebir como un instrumento de transformación y desarrollo. Es necesario insistir en los objetivos de la política industrial y en la movilización de los protagonistas de la vida económica y de las instituciones públicas y privadas para poner en marcha la inmensa tarea de la coordinación. En un régimen macroeconómico caracterizado por grandes restricciones, pero las empresas revelan un enorme dinamismo y las instituciones públicas y privadas siempre han actuado en forma creativa, es fundamental que la política industrial, recurra a las competencias empresariales e institucionales para la ingeniosa arquitectura de la coordinación.

Se trata de un desafío de persistencia, de construcción paulatina, de seguimiento, revisión y redefinición, y en la cual la gerencia de las empresas tienen el reto de responder positivamente con la redefinición de esquemas organizacionales y productivos generadores de competitividad a las empresas competir en el ámbito internacional con bienes y servicios aportadores de valor agregado y cuya demanda no se vea limitada en el mercado mundial. Saber entender e internalizar en la empresa el impacto de las políticas macroeconómicas y de factores meso económicos asociados a la competitividad es un punto de partida importante para las políticas macroeconómicas y de desarrollo productivo tengan un verdadero impacto a partir de las características de los bienes y servicios transados en el mundo.

\section{Referencias bibliográficas}

Baltagi, Badi (2005), Econometric Anaysis of Panel Data. Chichester: John Waley \& Sons Ltd.

Chenery, Hollis y Syrquin, Moshe (1989), Patter of Development, 1950 to 1983 Segundo edición. Washington, USA: The Worl Bank.

Chenery, Hollis (1982), Industrialization and Growth: The experiencie of large countries. The World Bank. № 539 , Washington D.C., pp 1-43

Chenery, Hollis (1986), Patter of Development, 1950 to 1983, Oxford University Press. Washington D.C.

Chica, Ricardo (1982), Una descripción de la evolución de la estructura industrial colombiana 1958-1980. Revista de Desarrollo y Sociedad, Documento de trabajo $N^{\circ} 70$, Colombia, Centro de Estudios sobre Desarrollo Económico, Universidad de Los Andes, pp 22-124.

Cimoli, Mario, Pereira, Wellintong Porcile y Scatolin, Fábio (2011), Structural change, tecnology, and economic growth: Brazil and the CIBS in a comparative perspective. Economic Change and Restructuring, Vol. 44, $\mathrm{N}^{\circ}$ 1, pp 25-47.

Clavijo, Sergio, Vera, Alejandro y Fandiño, Alejandro (2012), La desindustrialización en Colombia análisis cuantitativo de sus determinantes. Primera edición. Bogotá: Asociación Nacional de Instituciones Financieras.

Echavarria, Juan (2001), Colombia en la década de los noventa: neoliberalismo 
y reformas estructurales en el trópico. Cuadernos de Economía, Vol. 20, № 34, Colombia, Universidad Nacional de Colombia, pp 57-102.

Garay, Luis (1998), Colombia: Estructura Industrial e Internacionalización 1967-1996. Colombia, Departamento Nacional de Planeación.

García, José (2002), Liberalización, cambio estructural y crecimiento económico en Colombia. Cuadernos de Economía, Vol. 21, № 36, Colombia, Universidad Nacional de Colombia, pp 190-244.

Malaver, Florentino (2007), Dinámica y transformaciones de la industria colombiana. Cuadernos de Economía, Vol. 31, № 36 , Colombia, Universidad Nacional de Colombia, pp 273-323.

Maldonado, Alexander (2010), La evolución del crecimiento industrial y trasformación productiva en Colombia 1970-2005. (Tesis inédita de maestría). Colombia, Universidad Nacional de Colombia, Facultad de Ciencias Económicas.

Mesa, Ramón, Restrepo, Diana, y Aguirre, Yenny (2008), Crisis externa, desaceleración de la economía colombiana en 2008-2009. Perfil de Coyuntura Económica. $\mathrm{N}^{\circ} 12, \mathrm{pp}$ 31-67.

Misas, Gabriel (2002), La ruptura de los 90. Del gradualismo al colapso. Primera edición. Colombia, Universidad Nacional de Colombia.

Moncayo, Edgardo (2011), Cambio estructural, crecimiento e industrialización en América Latina 1950-2005. (Tesis inédita de doctorado). Colombia, Universidad Nacional de Colombia, Facultad de Ciencias Económicas.

Ortiz, Carlos; Uribe, José y Vivas, Harvey (2009), Transformación industrial, Autonomía tecnológica y Crecimiento Económico: Colombia 1925-2005. Archivos de Economía, No 352 , Colombia, Departamento Nacional de Planeación, pp 1-57.

Prebisch, Raul (1986), El desarrollo económico de la América Latina y algunos de sus principales problemas. Revista CEPAL, Vol. 26, No 103, Santiago de Chile, pp 479-502.

Ramírez, Juan, y Núñez, Liliana (2000), Reformas, crecimiento, progreso técnico y empleo en Colombia. Cepal: Serie Reformas Económicas. № 59 , $1-49$

Schiavo-Campo, Salvatore (1978), The simple measurement of structural change: A note. Economic Record, Vol. 54, No 2, Washington D.C, pp 261-263.

Silva, Ester y Teixeira, Aurora (2008), Surveying structural change: seminar contribución and bibliometric account. Structural Change and Economic Dynamic, Vol. 19, $\mathrm{N}^{\circ} 4$, Portugal, Universidad de Porto, Facultad de Letras, pp 273-300.

Syrquin, Moshe (1988, April), Growth and Structural Change in Latin America since 1960: A Comparative Analysis. Economic Development and Cultural Change, Vol. 34, No 3 , Estados Unidos, Universidad de Chicago, pp 433-454.

Thirwall, Antony (2003), La naturaleza del crecimiento económico (primera edición). Ciudad de México: Fondo de cultura económica México.

Vera, Leonardo (2000), La evolución macroeconómica en Venezuela y Colombia en los 90: algunos retos para la armonización. Revista Banco Central de Venezuela, Vol. 14, $\mathrm{N}^{\circ} 1$ Venezuela, pp 101-148.

Vera, Leonardo (2009), Cambio estructural, desindustrialización y pérdida de productividad: evidencia para Venezuela. Cuadernos del Cendes Vol. 26, Nº 71, Venezuela, pp 89-115.

Vernon, Ruttan (1998), Theory the new growth theory and development economics: A survey. The Journal of Development Studies, Vol. 35, № 2, pp 1-26. 